\title{
A NOTE ON SEPARATING FAMILIES OF REPRESENTATIONS
}

\author{
BRUCE A. BARNES
}

\begin{abstract}
Conditions are given that imply that a separating family of unitary representations of a locally compact group $G$ lifts to a separating family of *-representations of $L^{\prime}(G)$.
\end{abstract}

1. Introduction. Let $G$ be a locally compact group. A collection of unitary representations $\mathscr{Q}$ of $G$ is a separating family for $G$ if, given $x \in G, x \neq e$ (the identity of $G$ ), there exists $\pi \in \mathcal{Q}$ such that $\pi(x) \neq I$ (the identity operator). A collection of *-representations $\mathscr{R}$ of $L^{1}(G)$ is a separating family for $L^{1}(G)$ if, given $f \in L^{\prime}(G), f \neq 0$, there exists $\pi \in \mathcal{R}$ such that $\pi(f) \neq 0$. In this note we consider the question: Given that a collection $\mathcal{U}$ of unitary representations of $G$ is a separating family for $G$, under what conditions is the collection (lifted in the usual fashion to $L^{\prime}(G)$ ) a separating family for $L^{\prime}(G)$ ?

Even in the simplest cases a separating family of unitary representations of $G$ need not lift to a separating family for $L^{1}(G)$. For example, consider the group $G=\{e, x\}$ where $e$ is the identity of $G$ and $x^{2}=e$. Let $\pi$ be the one-dimensional unitary representation given by $\pi(e)=1, \pi(x)=-1$. Then $\pi$ separates points on $G$, but if $f \in L^{\prime}(G)$ is defined by $f(e)=1, f(x)=1$, then $\pi(f)=0$. Therefore $\{\pi\}$ is not a separating family for $L^{1}(G)$. On the other hand, if $Q$ is a separating family of *-representations of $L^{1}(G)$, then the corresponding family of unitary representations is separating for $G$. To see this, suppose there exists $y \in G, y \neq e$, such that $\pi(y)=I$ for all $\pi \in \mathbb{Q}$. For $f \in L^{1}(G)$ let $f_{y}$ denote the function defined by $f_{y}(x)=f(y x), x \in G$. A straightforward computation shows that

$$
\pi\left(f_{y}\right)=\pi(f) \text { for all } \pi \in \mathbb{Q}, f \in L^{1}(G) \text {. }
$$

Now choose $f$ to be the characteristic function of an open neighborhood $U$ of $e$ such that $U$ and $y^{-1} U$ are disjoint. Then $f \neq f_{y}$, but $f$ and $f_{y}$ cannot be separated by $Q$.

In $\$ 3$ of [1], S. Grosser and M. Moskowitz prove that if $G$ has a separating family of finite dimensional unitary representations, then $L^{1}(G)$ has a separating family of finite dimensional *-representations. Their proof uses the fact that in this case there is a continuous group monomorphism of $G$ onto a subgroup of a compact group. This is a special argument that does not generalize to other interesting collections of unitary representations of $G$, such as the class of square integrable representations.

Received by the editors February 8, 1982.

1980 Mathematics Subject Classification. Primary 43A65.

Key words and phrases. Separating family of representations.

(c) 1983 American Mathematical Society 0002-9939/82/0000-0752/\$02.25 
We prove an elementary result that sheds light on the question above and has as corollaries: the theorem of Grosser and Moskowitz, an application to the family of square integrable representations, and an application to the family of representations that lift to finite dimensionally spanned representations of $L^{\prime}(G)$.

2. The main result. The designation unitary representation will automatically include the weak continuity of the representation. When $\pi$ is a unitary representation of $G$, then we also denote by $\pi$ the *-representation of $L^{1}(G)$ obtained via the usual lifting formula.

Let $\Gamma$ be a topological space. We denote by $C(\Gamma)$ the algebra of all complex-valued bounded continuous functions on $\Gamma$. If $\Delta \subset \Gamma, f \in C(\Gamma)$, we set

$$
\|f\|_{\Delta}=\sup \{|f(x)|: x \in \Delta\} \text {. }
$$

Now let $\Omega$ be a compact Hausdorff space. Assume that $A$ is a closed *-subalgebra of $C(\Omega)$ which contains the constants. For $\omega \in \Omega$, let

$$
\tilde{\omega}=\left\{\omega^{\prime} \in \Omega: f(\omega)=f\left(\omega^{\prime}\right) \text { for all } f \in A\right\} .
$$

It is shown in [4, p. 126] that $\tilde{\Omega}$ is a compact Hausdorff space in a natural topology, $\omega \rightarrow \tilde{\omega}$ is a continuous map of $\Omega$ onto $\tilde{\Omega}$, and $\tilde{f}$ is continuous for all $f \in A$ where

$$
\tilde{f}(\tilde{\omega})=f(\omega), \quad f \in A, \omega \in \Omega .
$$

[4, Theorem (3.2.16)] shows that $f \rightarrow \tilde{f}$ is an isometric *-isomorphism of $A$ onto $C(\tilde{\Omega})$. We use this notation and terminology in the Lemma below.

LemMA. Let $A$ be a closed selfadjoint algebra of functions which contains the constants, $A \subset C(G)$. Let $K$ be a nonempty compact subset of $\Omega$ such that $A$ separates the points of $K$. Given $g \in C(K)$ there exists $h \in A$ such that $\|h\|_{G} \leqslant 2\|g\|_{K}$ and $h(x)=g(x)$ for all $x \in K$.

Proof. Let $\Omega$ be the Stone-Čech compactification of $G$. We consider $G$ as a dense subset of $\Omega$ (via the homeomorphic embedding of $G$ into $\Omega$ ). Note that $K$ is a compact subset of $\Omega$. Also we consider $A$ as a closed subalgebra of $C(\Omega)$. Now $\omega \rightarrow \tilde{\omega}$ is a continuous map of $\Omega$ onto $\tilde{\Omega}$, so that $\tilde{K}$ is compact in $\tilde{\Omega}$. Since $\omega \rightarrow \tilde{\omega}$ is one-to-one on $K, K$ and $\tilde{K}$ are homeomorphic.

Given $g \in C(K) \approx C(\tilde{K})$, choose $\tilde{h} \in C(\tilde{\Omega})$ such that $\tilde{h}(x)=g(x)$ for all $x \in \tilde{K}$ and $\|\tilde{h}\|_{\tilde{\Omega}} \leqslant 2\|g\|_{K}$. Then there exists $h \in A$ such that $\|h\|_{G}=\|h\|_{\Omega}=\|\tilde{h}\|_{\tilde{\Omega}}$ and $h(\omega)=\tilde{h}(\tilde{\omega}), \omega \in \Omega$. Therefore

$$
h(x)=\tilde{h}(\tilde{x})=g(\tilde{x})=g(x), \quad x \in K .
$$

If $\pi$ is a unitary representation of $G$, then let $\bar{\pi}$ denote some fixed conjugate representation of $\pi$ as defined in [2, pp. 16, 17]. Any two conjugate representations of $\pi$ are equivalent [2, Theorem (27.26)]. Also, if $\bar{\xi}, \bar{\eta} \in H_{\pi}$ (the representation space of $\pi$ ), then there exists $\xi, \eta \in H_{\pi}$ such that

$$
(\bar{\pi}(x) \bar{\xi}, \bar{\eta})=(\overline{\pi(x) \xi, \eta}), \quad \text { all } x \in G .
$$

Thus if $\mathcal{Q}$ is a collection of unitary representations of $G$ such that whenever $\pi \in \mathcal{Q}$, then $\bar{\pi} \in \mathscr{U}$ (for some conjugate $\bar{\pi}$ of $\pi$ ), then the corresponding collection of coefficient functions on $G$ is selfadjoint (conjugate-closed). 
THEOREM. Assume that $\mathscr{U}_{0}=\left\{\pi_{\lambda}\right\}_{\lambda \in \Lambda}$ is a collection of unitary representations of $G$ such that:

(i) $\mathscr{Q}_{0}$ is a separating family for $G$;

(ii) $\pi \in \mathcal{Q}_{0} \Rightarrow \bar{\pi} \in \mathcal{Q}_{0}$; and

(iii) the trivial representation is in $\mathscr{U}_{0}$.

Let $\Re_{0}$ be the set of all representations of $L^{\prime}(G)$ of the form $\pi_{\lambda_{1}} \otimes \pi_{\lambda_{2}} \otimes \cdots \otimes \pi_{\lambda_{n}}$ where $\lambda_{1}, \ldots, \lambda_{n}$ is any choice of $n$ (possibly nondistinct) elements of $\Lambda, 1 \leqslant n<\infty$. Then $\mathcal{R}_{0}$ is a separating family for $L^{\prime}(G)$.

Proof. Let $A_{0}$ be the algebra of functions on $G$ generated by the set of all coefficient functions

$$
x \rightarrow(\pi(x) \xi, \eta), \quad \pi \in \mathcal{U}_{0}, \xi, \eta \in H_{\pi} .
$$

By (i)-(iii), $A_{0}$ is a selfadjoint subalgebra of $C(G)$ that contains the constants and separates the points of $G$. To prove the result it suffices to show that if $f \in L^{\prime}(G)$ and $\int_{G} h f d x=0$ for all $h \in A_{0}$, then $f=0$. Thus assume $\int_{G} h f d x=0$ for all $h \in A_{0}$.

Let $\varepsilon>0$ be arbitrary. Choose a compact set $K \subset G$ such that $\int_{G \backslash K}|f| d x<\varepsilon$. Let $A$ be the uniform closure of $A_{0}$. For all $h \in A$,

$$
\left|\int_{K} h f d x\right|=\left|\int_{G} h f d x-\int_{G \backslash} h f d x\right|=\left|\int_{G \backslash K} h f d x\right| \leqslant \varepsilon\|h\|_{G} .
$$

Denote by $\alpha_{K}$, the continuous linear functional on $C(K)$ given by $\alpha_{K}(g)=\int_{K} g f d x$, $g \in C(K)$. For a given $g \in C(K)$, choose $h \in A$ as in the Lemma, so that $h(x)=$ $g(x)$ for all $x \in K$ and $\|h\|_{G} \leqslant 2\|g\|_{K}$. Then

$$
\left|\alpha_{K}(g)\right|=\left|\int_{K} g f d x\right|=\left|\int_{K} h f d x\right| \leqslant \varepsilon\|h\|_{G} \leqslant 2 \varepsilon\|g\|_{K} .
$$

Since $\left\|\alpha_{K}\right\|=\int_{K}|f| d x$, we have

$$
\int_{G}|f| d x=\int_{G \backslash K}|f| d x+\int_{K}|f| d x \leqslant \varepsilon+2 \varepsilon=3 \varepsilon .
$$

Then as $\varepsilon$ is arbitrary, $f=0$.

We remark that the Theorem holds with the measure algebra $M(G)$ in place of $L^{1}(G)$. The proof of this more general result is essentially the same as the proof given above.

3. Applications. Let $\mathscr{U}_{0}$ be the collection of all finite dimensional unitary representations of $G$. Then $\mathcal{U}_{0}$ satisfies (i)-(iii) of the Theorem. In addition, if $\pi, \tau \in \mathcal{Q}_{0}$, then $\pi \otimes \tau \in \mathcal{Q}_{0}$, and $\pi \otimes \tau$ is a direct sum of irreducible finite dimensional unitary representations. Therefore the Theorem implies the following result of Grosser and Moskowitz.

COROLlARY 1 (GROSSER-MOSKOWITZ). If the collection of finite dimensional unitary representations is a separating family for $G$, then the collection of irreducible finite dimensional *-representations of $L^{1}(G)$ is a separating family for $L^{1}(G)$. 
A unitary representation $\pi$ of a unimodular group $G$ is square integrable if all the coefficient functions

$$
x \rightarrow(\pi(x) \xi, \eta), \quad \xi, \eta \in H_{\pi},
$$

are in $L^{2}(G)$. A result of R. Kunze and E. Stein (see [5, p. 356]) shows that the following two statements are equivalent:

(1) $\pi$ is square integrable;

(2) $\exists M>0$ such that

$$
\|\pi(f)\| \leqslant M\|f\|_{2} \quad \text { for all } f \in L^{1}(G) \cap L^{2}(G) .
$$

Now assume that $\pi$ and $\tau$ are square integrable unitary representations. By the result of Kunze and Stein there exist $P>0, Q>0$, such that

$$
\|\pi(f)\| \leqslant P\|f\|_{2}, \quad\|\tau(f)\| \leqslant Q\|f\|_{2}, \quad \text { all } f \in L^{1} \cap L^{2} .
$$

Thus, for $f \in L^{1} \cap L^{2},\|f\|_{2} \leqslant 1$,

$$
\|(\pi \otimes \tau)(f)\|=\|\pi(f) \otimes \tau(f)\| \leqslant\|\pi(f)\|\|\tau(f)\| \leqslant P Q .
$$

Thus $\pi \otimes \tau$ is square integrable by the Kunze-Stein result. Then by [3, Corollary, p. 459], $\pi \otimes \tau$ is a direct sum of irreducible square integrable representations. This implies the following application of the Theorem.

COROLlARY 2. If the collection of square integrable unitary representations is a separating family for $G$ ( $G$ unimodular), then the collection of irreducible square integrable *-representations plus the trivial representation is a separating family for $L^{\prime}(G)$.

A *-representation $\pi$ of $L^{1}(G)$ is finite dimensionally spanned (FDS) if the span of the vectors $\pi(f) \xi$, where $f \in L^{\prime}(G), \xi \in H_{\pi}$, and $\pi(f)$ has finite dimensional range, is dense in $H_{\pi}$; see G. Warner's book [5, p. 231]. It is easy to show that if $\pi$ and $\tau$ are *-representations of $L^{\prime}(G)$ which are FDS, then $\pi \otimes \tau$ is FDS. Also, it can be shown that an FDS *-representation of $L^{\prime}(G)$ is a direct sum of irreducible FDS *-representations. Finally if $\pi$ is FDS, then so is any conjugate $\bar{\pi}$. These remarks yield our final application.

COROLLARY 3. If the collection of unitary representations $\pi$ of $G$ such that $\pi$ is FDS on $L^{\prime}(G)$ is a separating family for $G$, then the set of irreducible FDS *-representations of $L^{\prime}(G)$ is a separating family for $L^{\prime}(G)$.

\section{REFERENCES}

1. S. Grosser and M. Moskowitz, Harmonic analysis on central topological groups, Trans. Amer. Math. Soc. 156 (1971), 419-454.

2. E. Hewitt and K. Ross, Abstract harmonic analysis. II, Springer-Verlag, Berlin, 1970.

3. R. Kunze, A note on square-integrable representations, J. Funct. Anal. 6 (1970), 454-459.

4. C. Rickart, General theory of Banach algebras, Van Nostrand. New York, 1960.

5. G. Warner, Harmonic analysis on semi-simple Lie groups. I, Springer-Verlag, Berlin, 1972.

Department of Mathematics, University of Oregon, Eugene, Oregon 97403

Current address: Department of Mathematics, University College of Swansea, Singleton Park, Swansea SA2 8PP. Wales, United Kingdom 